\title{
High Performance Liquid Chromatographic Optimization Study of Environmental Estrogens
}

\author{
Jingyao SHEN, Min XUE \\ Beijing Institute of Technology, Beijing, China
}

\begin{abstract}
Nine kinds of environmental estrogens including $\beta$-estradiol, estrone, mestranol, ethisterone, ethinyloestradiol, diethylstilbestrol, cortisone, estriol and hydrocortisone were determined using high performance liquid chromatography (HPLC). The determination conditions were optimized that ProntSIL 120-5-C18-ace-EPS column as stationary phase, methanol/water $(82.5 / 17.5$, v/v) as mobile phase and flow rate as $1.0 \mathrm{~mL} / \mathrm{min}$. These compounds could be base-line separated in 15 minutes. The commercially available milk spiked with the estrogens was performed as the real sample. The recovery rate was in the range of 92.2\%-103.7\% while the relative standard deviation (RSD) was less than $10 \%$.
\end{abstract}

KEYWORDS: HPLC, estrogen, milk

\section{INTRODUCTION}

Activation or inhibition of the function of the endocrine system occured when environmental estrogen entered human's body. Synthesis, release, transport, binding and metabolism of normal endocrine substances in vivo can be interference at the same time. Recently years, the research of environment estrogens has become hot topic, and reports of the harm effect of the estrogens to human body are of common occurrence. For example, estrone can lead to the damage of liver function and Myocardial Infarction; ethisterone is thought to increase the risk of an ectopic pregnancy; hydrocortisone can result in myocardial injury and cause many complications, including increasing pressure in the brain. Therefore, enviroment estrogens were banned strictly and can not be detected in food by China's agricultural sector in 2002. So, to establish a method for the rapid, simple, accurate determination of estrogens has great social significance.

Though there has been a lot of estrogen detection methods recently, High performance liquid chromatography (HPLC) begins to play a more and more important role in estrogens analysis. But in recent years, the number of stationary phases for HPLC rised rapidly. Up to now, more than 750 different bulk materials could be purchased on the market, and the number would be increased because of the furtherd evelopment.
In this study, nine kinds of environmental estrogens including $\beta$-estradiol, estrone, mestranol, ethisterone, ethinyloestradiol, diethylstilbestrol, cortisone, estriol and hydrocortisone were determined using high performance liquid chromatography (HPLC). We compared the separation effect of four kinds of chromatographic columns, and the determination conditions were optimized that ProntSIL 120-5-C18-ace-EPS column as stationary phase, methanol/water $(82.5 / 17.5, \mathrm{v} / \mathrm{v})$ as mobile phase and flow rate as $1.0 \mathrm{~mL} / \mathrm{min}$.

\section{EXPERIMENT}

\subsection{Materials}

Methanol and acetonitrilewas purchased from J.T.Baker (America), $\beta$-estradiol, estrone, mestranol, ethisterone, ethynylestradiol, diethylstillbetrol, cortisone, estriol and hydrocortisone were purchased by J\&K chemical (China), the deionized water was obtained from a water purification machine (Aquapro RM-220, China).

HPLC analysis was carried out on SHIMAZDU LC-20AT high performance liquid chromatograph (Japan) using a SIL-10AF autosampler and SPDM20A diode array detector (DAD). PromoSIL C18

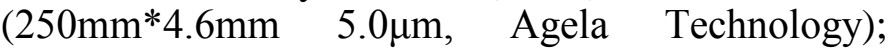
ProntSIL 120-5-C18-ace-EPS (250mm*4.6mm, $5.0 \mu \mathrm{m}$, BISCHOFF Germany), ProntSIL 120-5-C18AQ PLUS $\left(250 \mathrm{~mm}^{*} 4.6 \mathrm{~mm}, \quad 5.0 \mu \mathrm{m}\right.$, Agela Technology) and Thermo Hypersil Gold aQ 
$(250 \mathrm{~mm} * 4.6 \mathrm{~mm}, 5.0 \mu \mathrm{m}$, Thermo) were used in the study.

\subsection{Practical samples collection and treatment}

The pure milk samples were purchased in Walmart supermarket in Beijing. In the laboratory, the milk samples were kept in refrigerator retaining temperature $4{ }^{\circ} \mathrm{C}$ until the analysis. Acetonitrile/water $(1 / 1, \mathrm{v} / \mathrm{v})$ were added into $2 \mathrm{~mL}$ of milk samples. Vigorous shaking followed by sonication from 15 mins. The mixed sample was centrifuged (8000 rpm, 15mins) and the supernatant was collected and placed at a $15 \mathrm{~mL}$ centrifuge tube with $2 \mathrm{~mL}$ hexane. Standing and stratificated, then the upper solutionwas discarded. The lower solution was evaporated to dryness under a weak nitrogen stream at $40^{\circ} \mathrm{C}$. Then the residue was dissolved in $100 \mu \mathrm{L}$ of mobile phase. Before the analysis, samples were filtered with a $0.22 \mu \mathrm{m}$ nylon filter membrane.

\subsection{Standard solution preparation}

Nine estrogens were weighed accurately for $10 \mathrm{mg}$ respectively and placed into $25 \mathrm{~mL}$ volumetric flask. Subsequently, acetonitrile/water $(82.5 / 17.5, \mathrm{v} / \mathrm{v})$ was added with the concentration was $0.4 \mathrm{mg} / \mathrm{mL}$. The mixture was filtered by $0.45 \mu \mathrm{m}$ filter membrane and stored in refrigerator retaining $4^{\circ} \mathrm{C}$.

\section{RESULTS AND DISCUSSION}

\subsection{The choice of chromatographic conditions}

\subsubsection{Selection of detector and the detection wavelength.}

By scanning the ultraviolet absorption spectrum of 9 kinds of estrogen, acetylene progesterone, ethinylestradiol and diethylstilbestrol, cortisone, hydrocortisone had stronger absorption in the 220$260 \mathrm{~nm}$, while beta estradiol, estrone, estriol, estrone had stronger absorption in the $270-290 \mathrm{~nm}$, in order to achieve liquid chromatographic analysis of the nine compounds and ensure clear chromatograms, we choose our DAD detector, detection wavelength at $254 \mathrm{~nm}$ and $280 \mathrm{~nm}$, the two wavelengths of chromatogram has carried on the stack.

\subsubsection{Selection of chromatographic column.}

With four kinds of chromatographic column as stationary phase, methanol/water $(82.5 / 17.5, \mathrm{v} / \mathrm{v})$ as mobile phase, flow rate of $1.0 \mathrm{~mL} / \mathrm{min}$, detection wavelength of $254 \mathrm{~nm}$ and $280 \mathrm{~nm}$, column temperature stable at $30{ }^{\circ} \mathrm{C}$, taking nine kinds of estrogen mixture $10 \mu \mathrm{L}$ sample into test under the method of 2.3, the result is shown in figure 1 .

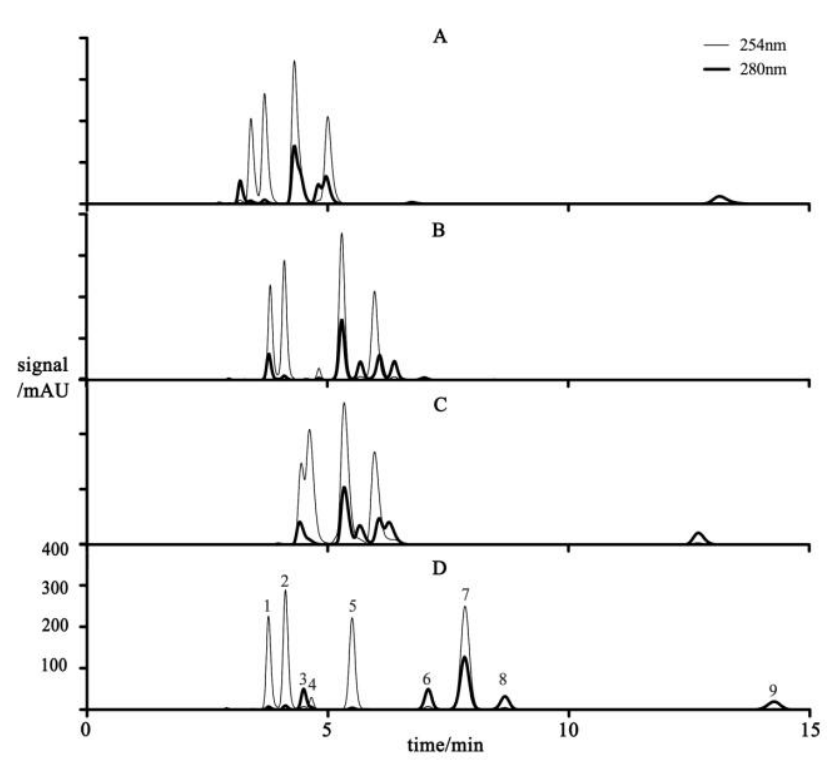

Figure 1 Result of the mixed standard estrogens solution on different chromatographic column chromatogram. (a) C18 column; (b) 120-5-C18-AQ PLUS column; (c) Hypersil Gold aQ column; (d)120-5-C18-ace-EPS column.

The results show that with C18, C18 - AQ PLUS and Hypersil Gold aQ column can't separate the nine estrogen baseline, while using C18 EPS column can complete the nine environmental estrogen baseline separation.

\subsubsection{The choice of mobile phase flow rate}

Velocity affects the size of the column system pressure, the degree of separation and elution time among these components. In order to further optimize the experiment, a 120-5 - EPS column as stationary phase, using C18 - ace - with the mobile phase at $1.2 \mathrm{~mL} / \mathrm{min}$ and $1.2 \mathrm{~mL} / \mathrm{min}$ of nine compounds are the prototype under two kinds of flow velocity in mixed solution, in order to achieve better separation effect, as shown in figure 2 .

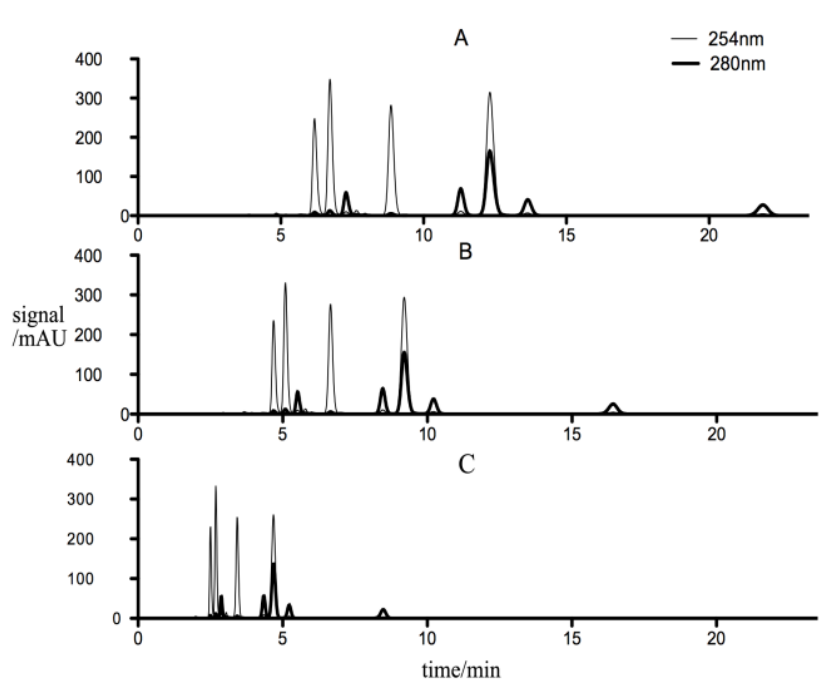

Figure 2 estrogen mixed standard solution in the chromatogram of different mobile phase $\mathrm{A}-\mathrm{C}$ was the separation results under flow rate of $0.6 \mathrm{ml} / \mathrm{min}, 1.0 \mathrm{ml} / \mathrm{min}, 1.2 \mathrm{ml} / \mathrm{min}$ condition respectively. 
According to the figure $1 \mathrm{D}$, figure $2 \mathrm{~A}, 2 \mathrm{~B}$ and the formula 1:

$$
R=\frac{2\left(t_{R_{2}} t_{R_{1}}\right)}{\left(W_{2}+W_{1}\right)}
$$

Compounds separation degree is calculated, of which the tR2 after one of the adjacent two peak retention time, tR 1 for two adjacent peak retention time of the top piece, $\mathrm{W} 1$ and $\mathrm{W} 2$ respectively two adjacent peak peak width, the calculation results were shown as table 1 .

Table 1 separation degree of nine kinds of estrogen

\begin{tabular}{|l|l|l|l|l|l|l|l|l|}
\hline & $1-2$ & $2-3$ & $3-4$ & $4-5$ & $5-6$ & $6-7$ & $7-8$ & $8-9$ \\
\hline $\begin{array}{c}\text { Degree of } \\
\text { separation }\end{array}$ & 1.7 & 1.5 & 1.5 & 3.5 & 5.0 & 2.0 & 2.5 & 5.5 \\
\hline
\end{tabular}

From table 1 shows, when the velocity was in the $1.0 \mathrm{~mL} / \mathrm{min}$, the compound isolation were greater than 1.5, to satisfy the demand of the "People's Republic of China pharmacopoeia" for HPLC separation degree, proving that this method to separate 9 kinds of estrogen can acheive very good effect.

\subsection{Detection limit and linear range}

Setting the mixture concentration as $0.001 \mathrm{mg} / \mathrm{ml}$, $0.005 \mathrm{mg} / \mathrm{ml}, 0.01 \mathrm{mg} / \mathrm{ml}, 0.05 \mathrm{mg} / \mathrm{ml}, 0.1 \mathrm{mg} / \mathrm{ml}$ of standard mixed solution. Using ProntSIL 120-5 C18 - ace - EPS column, mobile phase with 2.1.1 selected in the mobile phase, sample $10 \mu \mathrm{L}$, concentration of each sample three times respectively, chromatographic peak area average, nine compounds estrogen standard curve drawing, respectively, the abscissa for compound concentration, ordinate for high performance liquid chromatogram peak area, calculation of linear equation and correlation coefficient. Results show that the use of liquid phase under the condition of 9 kinds of estrogen in $0.001 \mathrm{mg} / \mathrm{mL} \sim 0.1 \mathrm{mg} / \mathrm{mL}$ concentration range inside sex is good. Results were shown in table 2 .

Table 2 Regression equation and Correlation coefficent

\begin{tabular}{|l|c|c|c|}
\hline Analytes & Regression equation & Linear interval & Correlation coefficent \\
\hline cortisone & $\mathrm{Y}=1.6 \times 10^{7} \mathrm{x}+1.0 \times 10^{4}$ & $0.001-0.1$ & $\mathrm{R}=1.000$ \\
\hline hydrocortisone & $\mathrm{Y}=4.1 \times 10^{7} \mathrm{x}+2.0 \times 10^{4}$ & $0.001-0.1$ & $\mathrm{R}=1.000$ \\
\hline estriol & $\mathrm{Y}=4.1 \times 10^{6} \mathrm{x}+3.9 \times 10^{3}$ & $0.001-0.1$ & $\mathrm{R}=1.000$ \\
\hline ethynylestradiol & $\mathrm{Y}=3.9 \times 10^{6} \mathrm{x}+4.6 \times 10^{3}$ & $0.001-0.1$ & $\mathrm{R}=0.999$ \\
\hline ethisterone & $\mathrm{Y}=2.2 \times 10^{7} \mathrm{x}+2.4 \times 10^{5}$ & $0.001-0.1$ & $\mathrm{R}=1.000$ \\
\hline estrone & $\mathrm{Y}=6.6 \times 10^{6} \mathrm{x}+2.5 \times 10^{4}$ & $0.001-0.1$ & $\mathrm{R}=0.999$ \\
\hline diethylstillbetrol & $\mathrm{Y}=3.0 \times 10^{7} \mathrm{x}+2.4 \times 10^{5}$ & $0.001-0.1$ & $\mathrm{R}=1.000$ \\
\hline$\beta$-estradiol & $\mathrm{Y}=4.4 \times 10^{6} \mathrm{x}+1.2 \times 10^{3}$ & $0.001-0.1$ & $\mathrm{R}=0.999$ \\
\hline mestranol. & $\mathrm{Y}=4.4 \times 10^{6} \mathrm{x}+5.5 \times 10^{3}$ & $0.001-0.1$ & $\mathrm{R}=1.000$ \\
\hline
\end{tabular}

\subsection{Recovery rate and precision}

We detected pretreatment milk sample under above HPLC detection condition, and we did not detect any estrogens. In order to determine the feasibility of this method in the actual samples test, the adding standard experiments was conducted. Three groups of blank milk samples were mixed with different concentrations of estrogen standard solution respectively. Recovery and RSD datas for the determination of nine kinds of estrogens in spiked milk were calculated using the formula 2 and the results were shown in table 3 .

$$
P=\frac{\left(C_{1} C_{2}\right)}{C_{3}} \quad 100 \%
$$

Table 3 Recovery and RSD for the determination of nine kinds of estrogens in spiked milk

\begin{tabular}{|l|c|c|c|c|c|c|c|c|c|}
\hline Analytes & $\begin{array}{c}\text { Spiked amount } \\
(\mathrm{mg} / \mathrm{mL})\end{array}$ & $\begin{array}{c}\text { Recovery } \\
(\%)\end{array}$ & $\begin{array}{c}\text { RSD } \\
(\%)\end{array}$ & $\begin{array}{c}\text { Spiked amount } \\
(\mathrm{mg} / \mathrm{mL})\end{array}$ & $\begin{array}{c}\text { Recovery } \\
(\%)\end{array}$ & $\begin{array}{c}\text { RSD } \\
(\%)\end{array}$ & $\begin{array}{c}\text { Spiked amount } \\
(\mathrm{mg} / \mathrm{mL})\end{array}$ & $\begin{array}{c}\text { Recovery } \\
(\%)\end{array}$ & $\begin{array}{c}\text { RSD } \\
(\%)\end{array}$ \\
\hline cortisone & 0.1 & 93.1 & 2.26 & 0.05 & 96.9 & 1.62 & 0.025 & 93.5 & 1.91 \\
\hline hydrocortisone & 0.1 & 93.3 & 1.91 & 0.05 & 101.6 & 2.03 & 0.025 & 100.1 & 1.78 \\
\hline estriol & 0.1 & 96.9 & 4.72 & 0.05 & 101.9 & 4.33 & 0.025 & 98.4 & 1.99 \\
\hline ethynylestradiol & 0.1 & 93.4 & 3.04 & 0.05 & 100.4 & 2.08 & 0.025 & 99.1 & 1.99 \\
\hline ethisterone & 0.1 & 93.9 & 1.84 & 0.05 & 100.5 & 3.31 & 0.025 & 100.1 & 2.01 \\
\hline estrone & 0.1 & 93.5 & 1.96 & 0.05 & 103.7 & 1.61 & 0.025 & 98.5 & 1.77 \\
\hline diethylstillbetrol & 0.1 & 94.2 & 2.03 & 0.05 & 98.6 & 1.53 & 0.025 & 98.5 & 1.76 \\
\hline$\beta$-estradiol & 0.1 & 92.2 & 2.11 & 0.05 & 95.5 & 1.79 & 0.025 & 97.7 & 1.56 \\
\hline mestranol & 0.1 & 92.4 & 2.07 & 0.05 & 100.1 & 1.91 & 0.025 & 89.3 & 1.49 \\
\hline
\end{tabular}


In table 3 , according to the results of standard addition of nine kinds of estrogens in milk recovery range from $92.2 \%$ to $103.7 \%$, relative standard deviation less than $10 \%$, the results are satisfactory.

\section{CONCLUSION}

We used ProntSIL 120-5 - C18 - ace - EPS chromatographic column and methanol/water $(82.5 / 17.5, \mathrm{v} / \mathrm{v})$ as mobile phase while setting flow rate of $1.0 \mathrm{~mL} / \mathrm{min}$, diode array detector was used at the same time. Results showed that nine kinds of estrogen including $\beta$-estradiol, estrone, mestranol, ethisterone, ethinyloestradiol, diethylstilbestrol, cortisone, estriol and hydrocortisone can be determined simultaneously within $15 \mathrm{~min}$, proving that high performance liquid chromatography (HPLC) method, the optimal chromatographic conditions can be put into use in the field of a variety of environmental estrogen detection. Meanwhile, this method is simple, high degree of separation, so it can be applied to analysis of environmental estrogen residues in milk and standards of environmental estrogen residues in dairy products.

\section{REFERENCES}

[1] LI Long-fei, ZHAO Xiao-lei, HE Jin-xing. Journal of Dairy Science and Technology, 2013(03): 24-27

[2] Eide M, Karlsen O A, Kryvi H, Olsvik P A,Goksoyr A, Aquatic toxicology (Amsterdam, Netherlands), 2014. 153: 110-115

[3] Nie M, Yang Y, Liu M, Yan C, Shi H, Dong W,Zhou J L, The Science of the total environment, 2014. 487: 785791

[4] Plotan M, Elliott C T, Frizzell C,Connolly L, Food Chemistry, 2014. 159: 157-165

[5] Jia J, Li Y, Lei Z, Hao Y, Wu Y, Zhao Q, Wang H, Ma L, Liu J, Zhao C, Jiang Y, Wang Y, Tan H, Dai X, Zhang W, Sun T,Yu J, Pharmaceutical Biology, 2013. 51(6): 744748

[6] Xiao X, Chen X, Xu X,Li G, Analytical Methods, 2013. 5(22): 6376-6381

[7] Pessoa G P, de Souza N C, Vidal C B, Alves J A C, Firmino P I M, Nascimento R F,Dos Santos A B, The Science of the total environment, 2014. 490: 288-295

[8] Barc J,Gregoraszczuk E L, Toxicology, 2014. 322: 14-22 